\title{
High prevalence of thalassemia in migrant populations in Guangdong Province, China
}

\author{
Bing Li ${ }^{1,2}$, Xiao-zhuang Zhang ${ }^{3}$, Ai-hua Yin ${ }^{4}$, Qing-guo Zhao ${ }^{3}$, Li Wu², Yuan-zhu Ma², Ming-yong Luo ${ }^{4}$ \\ and Shou-yi $\mathrm{Yu}^{1^{*}}$
}

\begin{abstract}
Background: The objectives of this study were to estimate the prevalence of thalassemia and to analyze the need for public health services for migrant populations in different cities in Guangdong Province, China.

Methods: A cross-sectional survey was conducted in 21 cities of Guangdong Province. Twenty-three types of a- and $\beta$-globin gene mutations were detected in a total of 14,230 pregnant women and 14,249 husbands.

Results: There was a $16.45 \%$ prevalence of thalassemia among the 28,479 individuals, and the prevalences of $\alpha-, \beta-$, and combined $\alpha-/ \beta$ - thalassemia were $12.03 \%, 3.80 \%$, and $0.63 \%$, respectively. Compared with the native city residents in the province, the migrants from within the province and the immigrants from outside the province had lower prevalences of thalassemia, but the prevalence values were $>11 \%$.

Conclusions: The prevalence values for thalassemia gene mutations were high in all three population groups studied in Guangdong Province. The results indicate that all segments of the Guangdong population should be screened for thalassemia.
\end{abstract}

Keywords: Thalassemia, Globin gene mutation, Prevention, Migrant population, Public health service

\section{Background}

Thalassemia is the most commonly inherited, recessive single gene disease. Globally, approximately $7 \%$ of pregnant women carry $\beta$ - or $\alpha$ zero-thalassemia, or other hemoglobin disorders [1,2], which imposes an enormous burden on families and society [3,4]. Thalassemia has become a public health problem in Guangdong Province, China. High prevalences of thalassemia are present in Guangdong populations; $17.70 \%$ of pregnant women, $15.94 \%$ of their husbands, and $16.03 \%$ of neonates are thalassemia carriers [5]. To prevent and control thalassemia major, the government of Guangdong has implemented the Thalassemia Prenatal Prevention and Control Program (TPPCP), which has been supported by annual funding of 35 million RMB since 2011. In

\footnotetext{
* Correspondence: pumpli587@sohu.com; 522276188@qq.com

${ }^{3}$ Department of the Office of the Dean, Guangdong Women and Children

Hospital, Guangzhou, 521 XingNan Street, Guangzhou, Guangdong 511442, China

${ }^{1}$ Department of Epidemiology, School of Public Health and Tropical Medicine, Southern Medical University, 1838 Guangzhou North Road, Guangzhou, Guangdong 510515, China

Full list of author information is available at the end of the article
}

2012, we used data obtained from this program and conducted a baseline survey on the epidemiological status of globin gene mutations in Guangdong.

As a developed area in China, Guangdong Province has a large amount of immigration between cities and between provinces, which imposes significant challenges for the public health services. Most public health services are only offered to native city residents who have a registered household as part of the household registry system. Little is known about the thalassemia prevalence in this and in other types of registered household populations in different cities in Guandong Province. We analyzed the $\alpha$ - and $\beta$-thalassemia prevalence in these populations.

\section{Methods}

The tested individuals were from one of three populations. The first population consisted of native city residents who were recorded in the local city household register. They had primary social and health insurance coverage and could obtain aid from the public health program. The second population consisted of migrants 
from within the province, and who were registered as residing in a household in another city in Guangdong Province. These residents had no primary social and health insurance coverage from the cities in which they were currently residing, and had non-transferable health insurance coverage from the cities or counties in which they were registered. They also could not obtain aid from the public health programs of the cities in which they were currently residing. The third population consisted of the immigrants from outside the province. These individuals were not registered as living in a household in Guangdong Province. They had no primary social and health insurance coverage in Guangdong, did not have transferable health coverage in the province in which they were registered, and could not obtain aid from the public health program in Guangdong.

This study used a cross-sectional survey design and was performed using data from the baseline TPPCP survey of Guangdong. The survey was conducted in the 21 cities of Guangdong Province. One district or county was randomly sampled from each city, and a total of 91 hospitals were selected according to the newborn delivery numbers in the sampled districts or counties. After informed consent was obtained, $3 \mathrm{~mL}$ peripheral vein blood (collected in an EDTA tube) was sampled from each pregnant woman and her husband during the delivery period. The samples were temporarily placed into a refrigerator at $4^{\circ} \mathrm{C}$, and then mailed on ice by express delivery to the genetics and metabolic diseases laboratory of Guangdong Women and Children Hospital for further analysis. They arrived at the laboratory within 3 days, where they were analyzed for the globin gene mutation. A total of 8,479 eligible blood samples were tested, which included 14,230 pregnant women and 14,249 husbands. The results for the number of samples from each city are presented in Table 1.

\section{Detection of $\alpha$ - and $\beta$-globin gene mutations}

Genomic DNA was extracted from all blood samples using a Lab-Aid 820 automation system (Zee San Biotech Company, Xiamen, Fujian, China). Twenty-three mutations of the a- and $\beta$-globin genes were identified using a Luminex 200 liquichip array system (R \& D Systems Inc., Minneapolis, MN, USA) [6,7]. The system was used throughout the process of probe design, the multiplex PCR, the attachment of probes to microspheres, and hybridization and analysis. The 23 thalassemia mutations included three $\alpha$-globin gene deletions (i.e., the Southeast Asian deletion [-SEA], the rightward deletion $[-\alpha 3.7]$, and the leftward deletion $[-\alpha 4.2])$. Three $\alpha$-globin gene point mutations were also analyzed (i.e., Hb Constant Spring, Hb Quong Sze, and Hb Westmead). The 17 $\beta$-globin gene point mutations were on codons $41 / 42$ (-TCTT), 654, -29 (A > G), -28 (A > G), codons 71/72
$(+\mathrm{A})$, codon $17(\mathrm{~A}>\mathrm{T})$, codon $43(\mathrm{G}>\mathrm{T}), \mathrm{Hb} \mathrm{E}(\beta 26[\mathrm{~B} 8]$ $\mathrm{Glu} \rightarrow$ Lys, GAG $>$ AAG or codon 26 [G $>$ A]), codons 27/ $28(+\mathrm{C})$, codon $31(-\mathrm{C}),-32(\mathrm{C}>\mathrm{A}),-30(\mathrm{~T}>\mathrm{C})$, codons 14/15 (+G), IVS-I-1 (G > T), IVS-I-5 (G > T), Int, and Cap.

\section{Statistical analysis}

We used the "vcd" and "car" program packages of R Version 3.0.1 statistical software (May 16, 2013, copyright 2013, R Foundation for Statistical Computing, Vienna, Austria) to analyze the data. We calculated the values for thalassemia carrier prevalence, with 95\% confidence intervals (95\% CIs), for the different household registrybased populations for all of the cities in Guangdong. The differences between prevalence values were compared using the chi-square test or the Mantel-Haenszel chi-square test. A thalassemia carrier was defined as an individual who carried any one of the 23 types of $\alpha$ and/or $\beta$-globin gene mutations.

\section{Results}

Blood samples were collected from a total of 28,479 individuals; $71.45 \%(20,349 / 28,479)$ were native residents of the city in which they resided, $19.83 \%(5,647 / 28,479)$ of them were migrants from another city within the province, and $8.72 \%(2,483 / 28,479)$ of them were immigrants from other provinces.

The results for the prevalence values for the different thalassemia genotypes are presented in Table 2. The overall prevalence of thalassemia carriers was $16.45 \%$. The prevalence of $\alpha-, \beta-$, and combined $\alpha-/ \beta$ - thalassemia carriers was $12.03 \%, 3.80 \%$, and $0.63 \%$, respectively. The specific prevalence values for the different clinical forms of the disease were also calculated. The prevalence of $\alpha^{+}$-thalassemia was 5.71\%, $\alpha^{0}$-thalassemia was $6.12 \%$, hemoglobin $\mathrm{H}(\mathrm{HbH})$ disease was $0.20 \%, \beta$ thalassemia minor $\left(\beta^{0} / \beta^{\mathrm{N}}\right.$ or $\left.\beta^{+} / \beta^{\mathrm{N}}\right)$ was $3.78 \%, \beta$ thalassemia intermedia was $0.01 \%, \alpha^{0}$ - or $\alpha^{+}$-thalassemia with $\beta$-thalassemia minor was $0.60 \%, \mathrm{HbH}$ disease with $\beta$-thalassemia minor was $0.02 \%, \alpha^{0}$-thalassemia with $\beta$ thalassemia intermedia carrier was $0.004 \%$. The top three genotypes of $\alpha$-thalassemia were $-{ }^{\mathrm{SEA}} / \alpha \alpha, \alpha^{3.7} \alpha /$ $\alpha \alpha$, and $\alpha^{4.2} \alpha / \alpha \alpha$. The top three genotypes of $\beta$ thalassemia were $\beta^{41-42} / \beta^{N}, \beta^{654} / \beta^{N}$, and $\beta^{-28} / \beta^{N}$.

The results for thalassemia carrier prevalence in different population groups are presented in Table 1 . The values for thalassemia carrier prevalence for the native city residents, the migrants from within the province, and the immigrants from outside the province were $17.16 \%$ (3491/20349), 16.04\% (906/5647), and 11.64\% (289/2483), respectively. Compared with the native city residents, the migrants from within the province and the immigrants from outside the province had a lower prevalence of thalassemia carriers, but the prevalence 
Table 1 The prevalence (\%) of thalassemia carriers among different household register population of cities of Guangdong, China

\begin{tabular}{|c|c|c|c|c|c|c|c|c|c|c|c|c|c|}
\hline \multirow{2}{*}{$\begin{array}{l}\text { Sampled cites } \\
\text { of Guangdong }\end{array}$} & \multicolumn{3}{|c|}{ Native city residents in the province } & \multicolumn{3}{|c|}{ Migrants from within the province } & \multicolumn{3}{|c|}{ Immigrants from outside the province } & \multicolumn{3}{|c|}{ Total } & \multirow{2}{*}{$\begin{array}{l}\text { Statistic } \\
\text { values }\end{array}$} \\
\hline & $\begin{array}{c}\text { Tested } \\
\text { individuals }\end{array}$ & $\begin{array}{l}\text { Thalassemia } \\
\text { carriers }\end{array}$ & $\begin{array}{c}\text { Prevalence } \\
\text { (\%95 CI) }\end{array}$ & $\begin{array}{c}\text { Tested } \\
\text { individuals }\end{array}$ & $\begin{array}{l}\text { Thalassemia } \\
\text { carriers }\end{array}$ & $\begin{array}{c}\text { Prevalence } \\
\text { (\%95 Cl) }\end{array}$ & $\begin{array}{c}\text { Tested } \\
\text { individuals }\end{array}$ & $\begin{array}{l}\text { Thalassemia } \\
\text { carriers }\end{array}$ & $\begin{array}{c}\text { Prevalence } \\
\text { (\%95 Cl) }\end{array}$ & $\begin{array}{c}\text { Tested } \\
\text { individuals }\end{array}$ & $\begin{array}{c}\text { Thalassemia } \\
\text { carriers }\end{array}$ & $\begin{array}{c}\text { Prevalence } \\
(\% 95 \mathrm{Cl})\end{array}$ & \\
\hline \multicolumn{14}{|l|}{ East Area } \\
\hline Chaozhou & 737 & 84 & 11.40 & 48 & 4 & 8.33 & 202 & 15 & 7.43 & 987 & 103 & 10.44 & $x^{2}=2.91$ \\
\hline Raoping & & & $(9.11,13.68)$ & & & $(0.55,16.11)$ & & & $(3.83,11.02)$ & & & $(8.54,12.33)$ & $P>0.05$ \\
\hline Jieyang & 1318 & 135 & 10.24 & 108 & 12 & 11.11 & 54 & 5 & 9.26 & 1480 & 152 & 10.27 & $x^{2}=0.14$ \\
\hline Puning & & & $(8.61,11.87)$ & & & $(5.21,17.01)$ & & & $(1.57,16.95)$ & & & $(8.73,11.81)$ & $P>0.05$ \\
\hline Shantou & 620 & 46 & 7.42 & 800 & 76 & 9.50 & 81 & 9 & 11.11 & 1501 & 131 & 8.73 & $x^{2}=2.51$ \\
\hline Jinping & & & $(5.37,9.47)$ & & & $(7.48,11.52)$ & & & $(4.30,17.92)$ & & & $(7.31,10.15)$ & $P>0.05$ \\
\hline Shanwei & 1118 & 123 & 11.00 & 60 & 9 & 15.00 & 51 & 4 & 7.84 & 1229 & 136 & 11.07 & $x^{2}=1.49$ \\
\hline Haifeng & & & $(9.18,12.83)$ & & & $(6.01,23.99)$ & & & $(0.50,15.18)$ & & & $(9.32,12.81)$ & $P>0.05$ \\
\hline \multirow[t]{2}{*}{ Total } & 3793 & 388 & 10.23 & 1016 & 101 & 9.94 & 388 & 33 & 8.51 & 5197 & 522 & 10.04 & $x^{2}=1.17$ \\
\hline & & & $(9.27,11.19)$ & & & $(8.11,11.77)$ & & & $(5.74,11.27)$ & & & $(9.23,10.86)$ & $P>0.05$ \\
\hline \multicolumn{14}{|l|}{ Montain Area } \\
\hline Heyuan & 1346 & 265 & 19.69 & 136 & 29 & 21.32 & 91 & 15 & 16.48 & 1573 & 309 & 19.64 & $x^{2}=0.82$ \\
\hline District & & & $(17.57,21.80)$ & & & $(14.47,28.17)$ & & & $(8.90,24.07)$ & & & $(17.69,21.60)$ & $P>0.05$ \\
\hline Meizhou & 934 & 199 & 21.31 & 26 & 5 & 19.23 & 42 & 8 & 19.05 & 1002 & 212 & 21.16 & $x^{2}=0.18$ \\
\hline Xingning & & & $(18.69,23.92)$ & & & $(4.16,34.30)$ & & & $(7.23,30.86)$ & & & $(18.64,23.67)$ & $P>0.05$ \\
\hline Qingyuan & 1165 & 237 & 20.34 & 88 & 15 & 17.05 & 92 & 16 & 17.39 & 1345 & 268 & 19.93 & $x^{2}=0.96$ \\
\hline Yingde & & & $(18.04,22.64)$ & & & $(9.23,24.86)$ & & & $(9.69,25.10)$ & & & $(17.80,22.05)$ & $P>0.05$ \\
\hline Shaoguan & 981 & 185 & 18.86 & 164 & 20 & 12.20 & 139 & 17 & 12.23 & 1284 & 222 & 17.29 & $x^{2}=7.15$ \\
\hline District & & & $(16.42,21.29)$ & & & $(7.21,17.18)$ & & & $(6.81,17.65)$ & & & $(15.23,19.35)$ & $P<0.05$ \\
\hline Yunfu & 1048 & 265 & 25.29 & 295 & 67 & 22.71 & 95 & 11 & 11.58 & 1438 & 343 & 23.85 & $x^{2}=9.28$ \\
\hline Luoding & & & $(22.67,27.90)$ & & & $(17.96,27.47)$ & & & $(5.18,17.98)$ & & & $(21.66,26.04)$ & $P<0.01$ \\
\hline \multirow[t]{2}{*}{ Total } & 5474 & 1151 & 21.03 & 709 & 136 & 19.18 & 459 & 67 & 14.60 & 6642 & 1354 & 20.39 & $x^{2}=11.50$ \\
\hline & & & $(19.95,22.10)$ & & & $(16.30,22.07)$ & & & $(11.38,17.81)$ & & & $(19.42,21.35)$ & $P<0.05$ \\
\hline \multicolumn{14}{|c|}{ The Pearl River Delta } \\
\hline Dongguan & 959 & 130 & 13.56 & 412 & 76 & 18.45 & 111 & 6 & 5.41 & 1482 & 212 & 14.30 & $x^{2}=13.38$ \\
\hline District & & & $(11.40,15.71)$ & & & $(14.72,22.17)$ & & & $(1.22,9.59)$ & & & $(12.53,16.08)$ & $P<0.01$ \\
\hline Foshan & 847 & 123 & 14.52 & 466 & 93 & 19.96 & 100 & 11 & 11.00 & 1413 & 227 & 16.07 & $x^{2}=8.63$ \\
\hline Nanhai & & & $(12.16,16.88)$ & & & $(16.35,23.57)$ & & & $(4.90,17.10)$ & & & $(14.16,17.97)$ & $P<0.05$ \\
\hline Guangzhou & 756 & 93 & 12.30 & 375 & 64 & 17.07 & 96 & 9 & 9.38 & 1227 & 166 & 13.53 & $x^{2}=6.40$ \\
\hline Panyu & & & $(9.97,14.63)$ & & & $(13.28,20.86)$ & & & $(3.57,15.18)$ & & & $(11.62,15.43)$ & $P<0.05$ \\
\hline Huizhou & 854 & 170 & 19.91 & 502 & 69 & 13.75 & 118 & 9 & 7.63 & 1474 & 248 & 16.82 & $x^{2}=16.33$ \\
\hline
\end{tabular}


Table 1 The prevalence (\%) of thalassemia carriers among different household register population of cities of Guangdong, China (Continued)

\begin{tabular}{|c|c|c|c|c|c|c|c|c|c|c|c|c|c|}
\hline District & & & $(17.24,22.57)$ & & & $(10.75,16.74)$ & & & $(2.86,12.39)$ & & & $(14.92,18.72)$ & $P<0.001$ \\
\hline Jiangmen & 1282 & 231 & 18.02 & 121 & 20 & 16.53 & 47 & 5 & 10.64 & 1450 & 256 & 17.66 & $x^{2}=1.81$, \\
\hline Xinhui & & & $(15.93,20.11)$ & & & $(9.94,23.11)$ & & & $(1.87,19.41)$ & & & $(15.70,19.61)$ & $P>0.05$ \\
\hline Shenzhen & 194 & 29 & 14.95 & 913 & 149 & 16.32 & 174 & 14 & 8.05 & 1281 & 192 & 14.99 & $x^{2}=7.85$ \\
\hline Baoan & & & $(9.96,19.94)$ & & & $(13.93,18.70)$ & & & $(4.02,12.07)$ & & & $(13.04,16.93)$ & $P<0.05$ \\
\hline Zhaoqing & 1143 & 215 & 18.81 & 191 & 46 & 24.08 & 85 & 15 & 17.65 & 1419 & 276 & 19.45 & $x^{2}=3.09$ \\
\hline Sihui & & & $(16.56,21.06)$ & & & $(18.05,30.12)$ & & & $(9.58,25.71)$ & & & $(17.40,21.50)$ & $P>0.05$ \\
\hline Zhongshan & 705 & 85 & 12.06 & 174 & 32 & 18.39 & 569 & 65 & 11.42 & 1448 & 182 & 12.57 & $x^{2}=6.21$ \\
\hline District & & & $(9.67,14.45)$ & & & $(12.66,24.12)$ & & & $(8.82,14.02)$ & & & $(10.87,14.27)$ & $P<0.05$ \\
\hline Zhuhai & 675 & 115 & 17.04 & 382 & 72 & 18.85 & 106 & 15 & 14.15 & 1163 & 202 & 17.37 & $x^{2}=1.40$ \\
\hline Doumen & & & $(14.22,19.86)$ & & & $(14.95,22.75)$ & & & $(7.55,20.75)$ & & & $(15.20,19.54)$ & $P>0.05$ \\
\hline \multirow[t]{2}{*}{ Total } & 7415 & 1191 & 16.06 & 3536 & 621 & 17.56 & 1406 & 149 & 10.60 & 12357 & 1961 & 15.87 & $x^{2}=37.06$ \\
\hline & & & $(15.23,16.89)$ & & & $(16.31,18.81)$ & & & $(9.00,12.20)$ & & & $(15.23,16.51)$ & $P<0.001$ \\
\hline \multicolumn{14}{|l|}{ West Area } \\
\hline Maoming & 1292 & 240 & 18.58 & 47 & 5 & 10.64 & 75 & 10 & 13.33 & 1414 & 255 & 18.03 & $x^{2}=3.1$ \\
\hline Huazhou & & & $(16.47,20.69)$ & & & $(1.87,19.41)$ & & & $(5.68,20.99)$ & & & $(16.04,20.03)$ & $P>0.05$ \\
\hline Yangjiang & 1393 & 355 & 25.48 & 74 & 4 & 5.41 & 54 & 8 & 14.81 & 1521 & 367 & 24.13 & $x^{2}=18.13$ \\
\hline Yangchun & & & $(23.21,27.76)$ & & & $(0.28,10.53)$ & & & $(5.39,24.24)$ & & & $(21.99,26.27)$ & $P<0.001$ \\
\hline Zhanjiang & 982 & 166 & 16.90 & 265 & 39 & 14.72 & 101 & 22 & 21.78 & 1348 & 227 & 16.84 & $x^{2}=2.62$ \\
\hline Lianjiang & & & $(14.57,19.24)$ & & & $(10.47,18.96)$ & & & $(13.77,29.79)$ & & & $(14.85,18.83)$ & $P>0.05$ \\
\hline \multirow[t]{2}{*}{ Total } & 3667 & 761 & 20.75 & 386 & 48 & 12.44 & 230 & 40 & 17.39 & 4283 & 849 & 19.82 & $x^{2}=16.11$ \\
\hline & & & $(19.45,22.06)$ & & & $(9.16,15.71)$ & & & $(12.52,22.26)$ & & & $(18.63,21.01)$ & $P<0.001$ \\
\hline \multirow[t]{2}{*}{ All Guangdong } & 20349 & 3491 & 17.16 & 5647 & 906 & 16.04 & 2483 & 289 & 11.64 & 28479 & 4686 & 16.45 & $x^{2}=49.85$, \\
\hline & & & $(16.64,17.67)$ & & & $(15.09,17.00)$ & & & $(10.38,12.89)$ & & & $(16.03,16.88)$ & $P<0.001$ \\
\hline
\end{tabular}


Table 2 The prevalence (\%) of different genotypes of thalassemia among pregnant coupes of Guangdong, China ( $n=28479)$

\begin{tabular}{|c|c|c|c|c|c|c|c|c|c|c|c|}
\hline \multicolumn{3}{|c|}{ Individuals only with $a$-thalassemia } & \multicolumn{3}{|c|}{ Individuals only with $\beta$-thalassemia } & \multicolumn{6}{|c|}{ Individuals combined $\alpha-/ \beta$ - thalassemia carriers } \\
\hline Genotypes & Frequency & $\begin{array}{l}\text { Prevalence } \\
(\% 95 \mathrm{Cl})\end{array}$ & $\begin{array}{l}\text { Genotypes } \\
\text { (clinic forms) }\end{array}$ & Frequency & $\begin{array}{l}\text { Prevalence } \\
(\% 95 \mathrm{Cl})\end{array}$ & $\begin{array}{l}\text { Genotypes } \\
\text { (clinic forms) }\end{array}$ & Frequency & $\begin{array}{l}\text { Prevalence }(\% \\
95 \mathrm{Cl})\end{array}$ & $\begin{array}{l}\text { Genotypes } \\
\text { (clinic forms) }\end{array}$ & Frequency & $\begin{array}{c}\text { Prevalence } \\
\text { (\%95 Cl) }\end{array}$ \\
\hline $\begin{array}{l}a^{+}- \\
\text {thalassemia }\end{array}$ & 1626 & $\begin{array}{l}5.71 \%(5.44 \\
5.98)\end{array}$ & $\begin{array}{l}\beta \text {-thalassemia } \\
\text { minor }\end{array}$ & 1077 & $\begin{array}{l}3.78(3.56 \\
4.00)\end{array}$ & $\begin{array}{c}{ }_{-}^{\mathrm{SEA}} / \mathrm{aa}, \beta^{41-42} / \beta^{N} \\
\left(a^{0}, \beta^{0} / \beta^{N}\right)\end{array}$ & 34 & $0.12(0.08,0.16)$ & $\begin{array}{c}-^{\mathrm{SEA}} / \mathrm{a}^{3.7}, \beta^{41-42} / \beta^{N}(\mathrm{HbH}, \\
\left.\beta^{0} / \beta^{N}\right)\end{array}$ & 1 & $\begin{array}{c}0.00 \\
(0.00,0.01)\end{array}$ \\
\hline$a^{3.7} a / a a$ & 911 & $\begin{array}{l}3.20(3.00 \\
3.40)\end{array}$ & $\beta^{41-42} / \beta^{N}\left(\beta^{0} / \beta^{N}\right)$ & 415 & $\begin{array}{l}1.46(1.32 \\
1.60)\end{array}$ & $\begin{array}{c}-\mathrm{SEA} / a \mathrm{a}, \beta^{654} / \beta^{N} \\
\left(a^{0}, \beta^{0} / \beta^{N}\right)\end{array}$ & 25 & $0.09(0.05,0.12)$ & $\begin{array}{c}{ }_{-}^{\mathrm{SEA}} / \mathrm{aa}, \beta^{-28} / \beta^{\mathrm{IVS}-1}\left(a^{0},\right. \\
\left.\beta^{+} / \beta^{0}\right)\end{array}$ & 1 & $\begin{array}{c}0.00 \\
(0.00,0.01)\end{array}$ \\
\hline$a^{4.2} a / a a$ & 313 & $\begin{array}{l}1.10(0.98 \\
1.22)\end{array}$ & $\beta^{654} / \beta^{N}\left(\beta^{0} / \beta^{N}\right)$ & 281 & $\begin{array}{l}0.99(0.87 \\
1.10)\end{array}$ & $\begin{array}{c}a^{3.7} / a a_{1} \beta^{41-42} / \beta^{N} \\
\left(a^{+}, \beta^{0} / \beta^{N}\right)\end{array}$ & 20 & $0.07(0.04,0.10)$ & $\begin{array}{c}-\mathrm{SEA} / \alpha a, \beta^{-29} / \beta^{N} \\
\left(a^{0}, \beta^{+} / \beta^{N}\right)\end{array}$ & 1 & $\begin{array}{c}0.00 \\
(0.00,0.01)\end{array}$ \\
\hline$a^{w s} a / a a$ & 239 & $\begin{array}{l}0.84(0.73 \\
0.94)\end{array}$ & $\beta^{17} / \beta^{N}\left(\beta^{0} / \beta^{N}\right)$ & 95 & $\begin{array}{l}0.33(0.27 \\
0.40)\end{array}$ & $\begin{array}{c}a^{3.7} / a a, \beta^{-28} / \beta^{N} \\
\left(a^{+}, \beta^{+} / \beta^{N}\right)\end{array}$ & 11 & $0.04(0.02,0.06)$ & $\begin{array}{c}{ }^{\mathrm{SEA}} / \mathrm{aa}, \beta^{\mathrm{IVS}-5} / \beta^{N} \\
\left(a^{0}, \beta^{+} / \beta^{N}\right)\end{array}$ & 1 & $\begin{array}{c}0.00 \\
(0.00,0.01)\end{array}$ \\
\hline$a^{C S} a / a a$ & 83 & $\begin{array}{l}0.29(0.23 \\
0.35)\end{array}$ & $\beta^{71-72} / \beta^{N}\left(\beta^{0} / \beta^{N}\right)$ & 24 & $\begin{array}{l}0.08(0.05 \\
0.12)\end{array}$ & $\begin{array}{c}-\mathrm{SEA} / a \mathrm{a}, \beta^{-28} / \beta^{N} \\
\left(a^{0}, \beta^{+} / \beta^{N}\right)\end{array}$ & 9 & $0.03(0.01,0.05)$ & $\begin{array}{c}a^{3.7} / a^{C S} a_{1} \beta^{41-42} / \beta^{N}\left(a^{0}\right. \\
\left.\beta^{0} / \beta^{N}\right)\end{array}$ & 1 & $\begin{array}{c}0.00 \\
(0.00,0.01)\end{array}$ \\
\hline$a^{\mathrm{QS}} \mathrm{a} / \mathrm{aa}$ & 47 & $\begin{array}{l}0.17(0.12 \\
0.21)\end{array}$ & $\beta^{27-28} / \beta^{N}\left(\beta^{0} / \beta^{N}\right)$ & 19 & $\begin{array}{l}0.07(0.04 \\
0.10)\end{array}$ & $\begin{array}{c}a^{3.7} / a a, \beta^{654} / \beta^{N} \\
\left(a^{+}, \beta^{0} / \beta^{N}\right)\end{array}$ & 8 & $0.03(0.01,0.05)$ & $\begin{array}{c}a^{3.7} / a a_{1} \beta^{-29} / \beta^{N} \\
\left(a^{+}, \beta^{+} / \beta^{N}\right)\end{array}$ & 1 & $\begin{array}{c}0.00 \\
(0.00,0.01)\end{array}$ \\
\hline$a^{3.7} a / a^{4.2} a$ & 9 & $\begin{array}{l}0.03(0.01 \\
0.05)\end{array}$ & $\beta^{14-15} / \beta^{N}\left(\beta^{0} / \beta^{N}\right)$ & 10 & $\begin{array}{l}0.04(0.01 \\
0.06)\end{array}$ & $\begin{array}{c}a^{\text {WS }} a / a a, \beta^{41-42} / \beta^{N}\left(a^{+},\right. \\
\left.\beta^{0} / \beta^{N}\right)\end{array}$ & 7 & $0.02(0.01,0.04)$ & $\begin{array}{c}a^{3.7} / a \alpha, \beta^{I V S-1} / \beta^{N} \\
\left(a^{+}, \beta^{0} / \beta^{N}\right)\end{array}$ & 1 & $\begin{array}{c}0.00 \\
(0.00,0.01)\end{array}$ \\
\hline$a^{3.7} a / a^{3.7} a$ & 8 & $\begin{array}{l}0.03(0.01 \\
0.05)\end{array}$ & $\beta^{43} / \beta^{N}\left(\beta^{0} / \beta^{N}\right)$ & 6 & $\begin{array}{l}0.02(0.00 \\
0.04)\end{array}$ & $\begin{array}{c}a^{4.2} / a a_{1} \beta^{41-42} / \beta^{N} \\
\left(a^{+}, \beta^{0} / \beta^{N}\right)\end{array}$ & 6 & $0.02(0.00,0.04)$ & $\begin{array}{c}a^{C S} a / a a_{1} \beta^{17} / \beta^{N} \\
\left(a^{+}, \beta^{0} / \beta^{N}\right)\end{array}$ & 2 & $\begin{array}{c}0.01 \\
(0.00,0.02)\end{array}$ \\
\hline$a^{3.7} a / a^{W S} a$ & 5 & $\begin{array}{l}0.02(0.00 \\
0.03)\end{array}$ & $\beta^{I V S-1} / \beta^{N}\left(\beta^{0} / \beta^{N}\right)$ & 6 & $\begin{array}{l}0.02(0.00 \\
0.04)\end{array}$ & $\begin{array}{c}{ }^{\mathrm{SEA}} / \mathrm{aa}, \beta^{17} / \beta^{N}\left(a^{0}\right. \\
\left.\beta^{0} / \beta^{N}\right)\end{array}$ & 4 & $0.01(0.00,0.03)$ & $\begin{array}{c}a^{c S} a / a a_{1} \beta^{654} / \beta^{N} \\
\left(a^{+}, \beta^{0} / \beta^{N}\right)\end{array}$ & 2 & $\begin{array}{c}0.01 \\
(0.00,0.02)\end{array}$ \\
\hline$a^{3.7} a / a^{C S} a$ & 3 & $\begin{array}{l}0.01(0.00 \\
0.02)\end{array}$ & $\beta^{\operatorname{lnt}} / \beta^{N}\left(\beta^{0} / \beta^{N}\right)$ & 1 & $\begin{array}{l}0.00(0.00 \\
0.01)\end{array}$ & $\begin{array}{c}-{ }^{\mathrm{SEA}} / a a_{1}, \beta^{\mathrm{E}} / \beta^{N}\left(a^{0},\right. \\
\left.\beta^{+} / \beta^{N}\right)\end{array}$ & 4 & $0.01(0.00,0.03)$ & $\begin{array}{c}a^{3.7} / a a_{1}, \beta^{1 \mathrm{VS}-5} / \beta^{N} \\
\left(a^{+}, \beta^{+} / \beta^{N}\right)\end{array}$ & 1 & $\begin{array}{c}0.00 \\
(0.00,0.01)\end{array}$ \\
\hline$a^{3.7} a / a^{Q S} a$ & 3 & $\begin{array}{l}0.01(0.00 \\
0.02)\end{array}$ & $\beta^{-28} / \beta^{N}\left(\beta^{+} / \beta^{N}\right)$ & 155 & $\begin{array}{l}0.54(0.46 \\
0.63)\end{array}$ & $\begin{array}{c}a^{3.7} / a a_{1} \beta^{17} / \beta^{N}\left(a^{+},\right. \\
\left.\beta^{0} / \beta^{N}\right)\end{array}$ & 4 & $0.01(0.00,0.03)$ & $\begin{array}{c}a^{4.2} / a a, \beta^{71-72} / \beta^{N} \\
\left(a^{+}, \beta^{0} / \beta^{N}\right)\end{array}$ & 1 & $\begin{array}{c}0.00 \\
(0.00,0.01)\end{array}$ \\
\hline$a^{4.2} a / a^{w s} a$ & 3 & $\begin{array}{l}0.01(0.00 \\
0.02)\end{array}$ & $\beta^{E} / \beta^{N}\left(\beta^{+} / \beta^{N}\right)$ & 24 & $\begin{array}{l}0.08(0.05 \\
0.12)\end{array}$ & $\begin{array}{c}a^{4.2} / a a, \beta^{-28} / \beta^{N} \\
\left(a^{+}, \beta^{+} / \beta^{N}\right)\end{array}$ & 4 & $0.01(0.00,0.03)$ & $\begin{array}{c}a^{4.2} / a a, \beta^{\mathrm{Cap}} / \beta^{N} \\
\left(a^{+}, \beta^{+} / \beta^{N}\right)\end{array}$ & 1 & $\begin{array}{c}0.00 \\
(0.00,0.01)\end{array}$ \\
\hline$a^{4.2} a / a^{C S} a$ & 1 & $\begin{array}{l}0.00(0.00 \\
0.01)\end{array}$ & $\beta^{\mathrm{Cap}} / \beta^{N}\left(\beta^{+} / \beta^{N}\right)$ & 23 & $\begin{array}{l}0.08(0.05 \\
0.11)\end{array}$ & $\begin{array}{c}a^{C S} a / a a, \beta^{41-42} / \beta^{N} \\
\left(a^{+}, \beta^{O} / \beta^{N}\right)\end{array}$ & 4 & $0.01(0.00,0.03)$ & $\begin{array}{c}a^{C S} a / a a_{1}, \beta^{-28} / \beta^{N} \\
\left(a^{+}, \beta^{+} / \beta^{N}\right)\end{array}$ & 1 & $\begin{array}{c}0.00 \\
(0.00,0.01)\end{array}$ \\
\hline$a^{4.2} a / a^{Q S} a$ & 1 & $\begin{array}{l}0.00(0.00 \\
0.01)\end{array}$ & $\beta^{-29} / \beta^{N}\left(\beta^{+} / \beta^{N}\right)$ & 17 & $\begin{array}{l}0.06(0.03 \\
0.09)\end{array}$ & $\begin{array}{c}a^{3.7} / a a, \beta^{71-72} / \beta^{N} \\
\left(a^{+}, \beta^{0} / \beta^{N}\right)\end{array}$ & 3 & $0.01(0.00,0.02)$ & $\begin{array}{c}a^{W S} a / a a_{,} \beta^{71-72} / \beta^{N}\left(a^{+},\right. \\
\left.\beta^{0} / \beta^{N}\right)\end{array}$ & 1 & $\begin{array}{c}0.00 \\
(0.00,0.01)\end{array}$ \\
\hline $\begin{array}{l}a^{0}- \\
\text { thalassemia }\end{array}$ & 1743 & $\begin{array}{l}6.12(5.84 \\
6.40)\end{array}$ & $\beta^{\text {IVS-5 }} / \beta^{N}\left(\beta^{+} / \beta^{N}\right)$ & 1 & $\begin{array}{l}0.00(0.00 \\
0.01)\end{array}$ & $\begin{array}{c}a^{4.2} / a a_{1} \beta^{17} / \beta^{N}\left(a^{+},\right. \\
\left.\beta^{0} / \beta^{N}\right)\end{array}$ & 3 & $0.01(0.00,0.02)$ & $\begin{array}{c}a^{w S} a / a a_{1}, \beta^{E} / \beta^{N} \\
\left(a^{+}, \beta^{+} / \beta^{N}\right)\end{array}$ & 1 & $\begin{array}{c}0.00 \\
(0.00,0.01)\end{array}$ \\
\hline$-{ }^{\mathrm{SEA}} / \mathrm{aa}$ & 1743 & $\begin{array}{l}6.12(5.84 \\
6.40)\end{array}$ & $\begin{array}{l}\beta \text {-thalassemia } \\
\text { intermedia }\end{array}$ & 4 & $\begin{array}{l}0.01(0.00 \\
0.03)\end{array}$ & $\begin{array}{c}a^{4.2} / a a, \beta 654 / \beta^{N} \\
\left(a^{+}, \beta^{0} / \beta^{N}\right)\end{array}$ & 3 & $0.01(0.00,0.02)$ & $\begin{array}{c}a^{\text {WS }} a / a a_{1}, \beta^{\text {IVS-1 }} / \beta^{N} \\
\left(a^{+}, \beta^{O} / \beta^{N}\right)\end{array}$ & 1 & $\begin{array}{c}0.00 \\
(0.00,0.01)\end{array}$ \\
\hline $\begin{array}{l}\mathrm{HbH} \\
\text { disease }\end{array}$ & 58 & $\begin{array}{l}0.20(0.15 \\
0.26)\end{array}$ & $\beta^{-28} / \beta^{E}\left(\beta^{+} / \beta^{+}\right)$ & 1 & $\begin{array}{l}0.00(0.00 \\
0.01)\end{array}$ & $\begin{array}{c}-\mathrm{SEA} / \mathrm{a}^{3.7}, \beta^{654} / \beta^{\mathrm{N}} \\
\left(\mathrm{HbH}, \beta^{0} / \beta^{N}\right)\end{array}$ & 2 & $0.01(0.00,0.02)$ & & & \\
\hline$-{ }^{\mathrm{SEA}} / \mathrm{a}^{3.7}$ & 33 & $\begin{array}{l}0.12(0.08 \\
0.16)\end{array}$ & $\beta^{41-42} / \beta^{\mathrm{Cap}}\left(\beta^{0} / \beta^{+}\right)$ & 1 & $\begin{array}{l}0.00(0.00 \\
0.01)\end{array}$ & $\begin{array}{c}{ }^{\mathrm{SEA}} / \mathrm{a}^{\mathrm{CS}} \mathrm{a}, \beta^{41-42} / \beta^{\mathrm{N}} \\
\left(\mathrm{HbH}, \beta^{0} / \beta^{N}\right)\end{array}$ & 2 & $0.01(0.00,0.02)$ & & & \\
\hline$-{ }^{\mathrm{SEA}} / a^{4.2}$ & 15 & $\begin{array}{l}0.05(0.03 \\
0.08)\end{array}$ & $\beta^{41-42} / \beta^{E}\left(\beta^{0} / \beta^{+}\right)$ & 1 & $\begin{array}{l}0.00(0.00 \\
0.01)\end{array}$ & $\begin{array}{c}{ }_{-}^{\mathrm{SEA}} / \mathrm{aa}, \beta^{71-72} / \beta^{N} \\
\left(a^{0}, \beta^{0} / \beta^{N}\right)\end{array}$ & 2 & $0.01(0.00,0.02)$ & & & \\
\hline
\end{tabular}


Table 2 The prevalence (\%) of different genotypes of thalassemia among pregnant coupes of Guangdong, China ( $\mathbf{n}=\mathbf{2 8 4 7 9 )}($ Continued)

\begin{tabular}{|c|c|c|c|c|c|c|c|c|c|}
\hline$-{ }^{\mathrm{SEA}} / a^{\mathrm{WS}} \mathrm{a}$ & 5 & $\begin{array}{c}0.02 \\
(0.00,0.03)\end{array}$ & $\beta^{-28} / \beta^{17}\left(\beta^{+} / \beta^{0}\right)$ & 1 & $\begin{array}{c}0.00(0.00 \\
0.01)\end{array}$ & $\begin{array}{c}a^{4.2} / a a_{1} \beta^{E} / \beta^{N} \\
\left(a^{+}, \beta^{+} / \beta^{N}\right)\end{array}$ & $0.01(0.00,0.02)$ & & \\
\hline$-{ }^{\mathrm{SEA}} / \mathrm{a}^{\mathrm{CS}} \mathrm{a}$ & 3 & $\begin{array}{c}0.01 \\
(0.00,0.02)\end{array}$ & & & & $\begin{array}{c}a^{W S} a / a a_{1} \beta^{654} / \beta^{N} \\
\left(a^{+}, \beta^{0} / \beta^{N}\right)\end{array}$ & $0.01(0.00,0.02)$ & & \\
\hline$-{ }^{\mathrm{SEA}} / \mathrm{a}^{\mathrm{QS}} \mathrm{a}$ & 2 & $\begin{array}{c}0.01 \\
(0.00,0.02)\end{array}$ & & & & $\begin{array}{c}{ }^{\mathrm{SEA}} / \alpha^{3.7}, \beta^{17} / \beta^{N} \\
\left(\mathrm{HbH}, \beta^{0} / \beta^{N}\right)\end{array}$ & $0.00(0.00,0.01)$ & & \\
\hline Total & 3427 & $\begin{array}{c}12.03 \\
(11.66,12.41)\end{array}$ & Total & 1081 & $\begin{array}{c}3.80 \\
(3.57,4.02)\end{array}$ & & Total & 178 & $\begin{array}{c}0.63 \\
(0.53,0.72)\end{array}$ \\
\hline
\end{tabular}

Notes: 1. $\mathrm{Cl}$ is Confidence Interval. 2. $\mathrm{Hb} \mathrm{H}$ disease means Hemoglobin $\mathrm{H}(\mathrm{HbH})$ disease. 3. $\beta^{\mathrm{N}}$ means normal $\beta$-globin allele gene. 
was $>11 \%$. The difference between these prevalence values was significant $\left(\chi^{2}=49.85, P<0.001\right)$.

For thalassemia carrier prevalence in the different areas of Guangdong Province, the differences between the different household registry populations were variable. Prevalence values were not significantly different $(P>0.05)$ between the four cities in the east area. In the mountain area, two of five cities had a significantly different prevalence $(P<0.05)$. In the Pearl River delta area, six of nine cities had a significantly different prevalence $(P<0.05)$, and in seven cities migrants from within the province had a higher prevalence of thalassemia carriers. In the west area, one of three cities had a significantly different prevalence value $(P<0.05)$.

\section{Discussion}

Our results indicated that thalassemia is a serious public health problem in Guangdong. The average prevalence of thalassemia in the Guangdong population was $16.45 \%$, and the prevalence of $\alpha-, \beta-$, and combined $\alpha-/ \beta-$ thalassemia was $12.03 \%, 3.80 \%$, and $0.63 \%$, respectively. These values were higher than values previously reported for prevalence for Guangdong Province $[8,9]$. Our data also indicated that the native city resident population in the province, the migrant population from within the province, and the immigrant population from outside the province also have had a high prevalence of thalassemia. In some surveyed cities, the thalassemia prevalence in the migrant population from within the province was higher compared with the prevalence in native city residents in the province. In all targeted populations, the native city residents and the immigrants from other cities of the same or other provinces belong to the same ethnic group, and the finding that there were similar prevalences of thalassemia mutations supports this observation.

Worldwide, 56,000 conceptions per year have a major thalassemia disorder. Approximately 30,000 of these are affected by $\beta$-thalassemia major, and 3,500 succumb from hydrops fetalis syndrome during the perinatal period [1]. Interestingly, in Guangdong, thalassemia also causes a significant economic and disease burden. Thalassemias were originally characteristic of the tropics and subtropics, but are now common worldwide because of migration $[10,11]$. Because thalassemia can be controlled in a cost-effective manner using programs that integrate treatment with carrier detection and genetic counseling, the World Health Organization has recommended global development of these services [12]. Many countries and areas have implemented antenatal or premarital thalassemia screening programs [13,14]. Since the 1990s, some hospitals have delivered voluntary antenatal thalassemia screening and counseling services in Guangdong Province [14]. In 2011, the Guangdong government initiated an antenatal thalassemia prevention and control program, which includes 35 million RMB per year in financial support.

Because of the household registry arrangement and resource limitations, public health services for thalassemia prevention have only been offered to native city residents in the province. However, our results indicate that all segments of the Guangdong population should be screened for thalassemia. The migrants from within the province and the immigrants from outside the province accounted for nearly $30 \%$ of the total population in many cities in Guandong, which could affect the outcomes of thalassemia prevention programs and challenge the public health service system of Guangdong. The results of a Thalassemia International Federation study indicated that countries with a strong prevention and treatment program are well prepared to face the problem of hemoglobinopathy gene migration into the population [14].

Previously, the thalassemia prevalence reported for Guangdong Province was approximately $5-11 \%[9,10]$. The differences between these figures and our results were mainly owing to differences in areas selected for sampling, the populations examined, the detection methods, and the spectra of globin gene mutations examined. We sampled a greater number of cities and populations. We used the more sensitive liquichip array system for detection of globin gene mutation and found 23 types of globin gene mutations. A higher prevalence of thalassemia carriers (24.51\%) has been reported for Guangxi Province [15]. Bangalore et al. reported that the prevalence of the $\beta$-thalassemia trait among pregnant women was $8.5 \%$, [16] which was also higher than that of Guangdong, but these results were estimated from data from an antenatal care clinic.

The analysis of our data revealed that $0.22 \%$ (64/ 28479) of individuals were carriers of $\mathrm{HbH}$ disease, which could be a serious health problem for pregnant couples. The incidence of $\mathrm{HbH}$ disease has been reported to be $0.43 \%$ in Guangxi Province [15]. $\mathrm{HbH}$ disease is predominantly seen in South East Asia, the Middle East, and the Mediterranean [17].

There are some limitations that must be taken into consideration in our study. We only sampled one county or district in each city, so the results might not represent the true prevalence of thalassemia carriers in the population of that city. We also did not consider the duration of residence of the migrant populations.

\section{Conclusion}

The prevalence of thalassemia gene mutations was high in native city resident populations in Guangdong Province, the migrant populations from within the province, and the immigrant populations from outside the 
province. The results indicate that all segments of the Guangdong population should be screened for thalassemia. The effectiveness of thalassemia prevention programs might be improved if the entire population in Guangdong Province were included. Policy makers should establish a strategy that allows affordable thalassemia prevention public health programs to be provided to inter-city and inter-province migrant populations in Guangdong Province.

\section{Ethical consideration}

Ethical approval was obtained from Guangdong Women and Children Hospital Ethics Committee.

\section{Competing interests}

The authors declare that they have no competing interests.

\section{Authors' contributions}

$\mathrm{BL}$ performed the statistical analysis and drafted the manuscript. BL, $X Z$, AYand QZ contributed to conception and design of the study and quality monitoring. AY, ML, LW, and M Y contributed to Lab test, Lab quality monitoring and edit the manuscript. SY contributed to instruction of study design and data analysis. All authors read and approved the final manuscript.

\section{Acknowledgments}

The authors would like to thank all the members of Guangdong thalassemia prevention and control program management team, and all the workers of data collection in all sampled hospitals.

The study was the program of the medical scientific research fund of Guangdong, the number were C2012009 and C2012010.

\section{Author details}

${ }^{1}$ Department of Epidemiology, School of Public Health and Tropical Medicine, Southern Medical University, 1838 Guangzhou North Road, Guangzhou, Guangdong 510515, China. ²Department of Healthcare, Guangdong Women and Children Hospital, Guangzhou, Guangdong, China. ${ }^{3}$ Department of the Office of the Dean, Guangdong Women and Children Hospital, Guangzhou, 521 XingNan Street, Guangzhou, Guangdong 511442 , China. ${ }^{4}$ Center of Prenatal Diagnosis, Guangdong Women and Children Hospital, Guangzhou, Guangdong, China.

Received: 7 March 2014 Accepted: 26 August 2014

Published: 2 September 2014

\section{References}

1. Modell B, Darlison M: Global epidemiology of haemoglobin disorders and derived service indicators. Bull World Health Organ 2008, 86(6):480-487.

2. Weatherall DJ: Thalassemia as a global health problem: recent progress toward its control in the developing countries. Ann N Y Acad Sci 2010, 1202:17-23

3. Suchdev PS, Ruth LJ, Marie E, Macharia A, Williams TN: The burden and consequences of inherited blood disorders among young children in western Kenya. Maternal and Child Nutrition 2012. doi:10.1111/j.17408709.2012.00454.x.

4. Arthorn Riewpaiboon, Issarang Nuchprayoon, Kitti T, Indaratna K, Thavorncharoensap M, Ubol BO: Economic burden of beta-thalassemia/Hb E and beta-thalassemia major in Thai children. BMC Research Notes 2010, 3:29.

5. Yin A, Li B, Luo M, Xu L, Wu L, Zhang L, Ma Y, Chen T, Gao S, Liang J, Guo H, Qin D, Wang J, Yuan T, Wang Y, Huang WW, He WF, Zhang Y, Liu C, Xia S, Chen Q, Zhao Q, Zhang X: The Prevalence and Molecular Spectrum of a- and b-Globin Gene Mutations in 14,332 Families of Guangdong Province, China. PLoS One 2014, 9(2):e89855. doi:10.1371/journal. pone.0089855.

6. Han X, Wang H, Chen H, Mei L, Wu S, Jia G, Cheng T, Zhu S, Lin X: Development and primary application of a fluorescent liquid bead array for the simultaneous identification of multiple genetically modified maize. Biosens Bioelectron 2013, 49:360-366. doi:10.1016/j.bios.2013.05.045. Epub 2013 Jun.

7. Spierings $G$, Dunbar SA: Pharmacogenetics using Luminex ${ }^{\circledR}$ xMAP $^{\circledR}$ technology: a method for developing a custom multiplex single nucleotide polymorphism mutation assay. Methods Mol Biol 2013, 1015:115-126. doi:10.1007/978-1-62703-435-7_7.

8. Zeng YT, Huang SZ: Disorders of haemoglobin in China. J Med Genet 1987, 24(10):578-583.

9. Xu XM, Zhou YQ, Luo GX, Liao C, Zhou M, Chen PY, Lu JP, Jia SQ, Xiao GF, Shen X, Li J, Chen HP, Xia YY, Wen YX, Mo QH, Li WD, Li YY, Zhuo LW, Wang ZQ, Chen YJ, Qin CH, Zhong M, 5: The prevalence and spectrum of alpha and beta thalassemia in Guangdong Province: implications for the future health burden and population screening. J Clin Pathol 2004, 57:517-522.

10. Weatherall DJ, Clegg JB: Inherited haemoglobin disorders: an increasing global health problem. Bull World Health Organ 2001, 79:704-712. pmid: 11545326.

11. Modella B, Darlisona M: Global epidemiology of haemoglobin disorders and derived service indicators. Bull World Health Organ 2008, 86:480-487.

12. Michalis A, Joan-Lluis Vives C, Soteriades ES, Eleftheriou A: The Impact of Migrations on the Health Services for Rare Diseases in Europe: The Example of Haemoglobin Disorders. The Scientific World Journal 2013. Article ID 727905, 10 pages, http://dx.doi.org/10.1155/2013/727905.

13. Antonio C, Yuet Wai K: The Prevention of Thalassemia. Cold Spring Harb Perspect Med 2012, 3:a011775.

14. Cousens NE, Gaff CL, Metcalfe SA, Delatycki MB: Carrier screening for Beta-thalassemia: a review of international practice. Eur J Hum Genet 2010, 1077-1083. doi:10.1038/ejhg.2010.90.

15. Xiong F, Sun M, Zhang X, Cai R, Zhou Y, Lou J, Zeng L, Sun Q, Xiao Q, Shang $X$, Wei $X$, Zhang T, Chen P, Xu X: Molecular epidemiological survey of haemoglobinopathies in the Guangxi Zhuang Autonomous Region of southern China. Clin Genet 2010, 78:139-148.

16. Praveen K, Ramesh Masthi NR, Niveditha SR, Suvarna R: The Prevalence of the Beta Thalassemia Trait among the Pregnant Women who attended the ANC Clinic in a PHC, by using the NESTROF Test in Bangalore, Karnataka. Journal of Clinical and Diagnostic Research 2013, 7(7):1414-1417.

17. Cornelis L: Harteveld, Douglas R Higgs. a-thalassaemia. Harteveld and Higgs. Orphanet J Rare Dis 2010, 5:13.

doi:10.1186/1471-2458-14-905

Cite this article as: Li et al.: High prevalence of thalassemia in migrant populations in Guangdong Province, China. BMC Public Health 2014 14:905.

\section{Submit your next manuscript to BioMed Central and take full advantage of:}

- Convenient online submission

- Thorough peer review

- No space constraints or color figure charges

- Immediate publication on acceptance

- Inclusion in PubMed, CAS, Scopus and Google Scholar

- Research which is freely available for redistribution 